\title{
Towards a broadband chirped pulse Fourier transform microwave spectrometer
}

\author{
D Mani ${ }^{1}$, V T Bhat ${ }^{2}, \mathrm{~K} J$ Vinoy $^{2}$ and E Arunan ${ }^{1}$ * \\ ${ }^{1}$ Department of Inorganic and Physical Chemistry, Indian Institute of Science, Bangalore 560012, India \\ ${ }^{2}$ Department of Electrical Communication Engineering, Indian Institute of Science, Bangalore 560012, India \\ Received: 18 July 2011 / Accepted: 07 March 2012 / Published online: 12 April 2012
}

\begin{abstract}
This article gives a brief review of microwave spectroscopy and the experimental techniques used for obtaining microwave spectrum of molecules and complexes since 1950s. It presents a brief summary of the pulsed nozzle Fourier transform microwave (PNFTMW) spectrometer, fabricated in our laboratory, and discusses some of the important results obtained using the spectrometer. The most significant among the results from this spectrometer is the direct structural determination of weakly bound complexes involving $\mathrm{H}_{2} \mathrm{O} / \mathrm{H}_{2} \mathrm{~S}$. These have challenged the conventional wisdom on hydrogen bonding leading us to propose a modern definition for the same through IUPAC. The limitations of the PNFTMW spectrometer and the need for the new chirped pulse Fourier transform microwave spectrometer are discussed as well. Moreover, preliminary results from our laboratory on generating a $1 \mu$ s chirped pulse of $1 \mathrm{GHz}$ bandwidth are given.
\end{abstract}

Keywords: Rotational spectroscopy; Supersonic beams; Chirp-pulse; Fourier-transform microwave spectrometer

PACS Nos.: $33.20 . B x ; 07.57 . P t ; 84.40 .-x$

\section{Introduction}

Microwave spectroscopy is widely regarded as the most accurate tool for structure determination of molecules in the gas phase. The frequencies corresponding to transition between different rotational energy levels depend on a molecule's moment of inertia (mass distribution) along the three principal axes of inertia. Thus by measurement of transition frequencies one can calculate the principal moment of inertia for the molecule. Isotopic substitution analysis gives the inter-atomic distances and angles for the molecule, eventually leading to its structure $[1,2]$. Molecules are divided into three groups according to the principal moment of inertia:

(i) Linear molecules $\left(I_{a}=0, I_{b}=I_{c}\right)$

(ii) Symmetric top molecules $\left(I_{a}<I_{b}=I_{c}\right.$ or $\left.I_{a}=I_{b}<I_{c}\right)$

(iii) Asymmetric top molecules $\left(I_{a}<I_{b}<I_{c}\right)$

*Corresponding author, E-mail: arunan@ipc.iisc.ernet.in
Here $I_{a}, I_{b}$ and $I_{c}$ are moments of inertia along the principal axes $a-c$ respectively. The convention for labeling the principal axes is such that $I_{a} \leq I_{b} \leq I_{c}$. Symmetric top molecules are further classified as prolate $\left(I_{a}<I_{b}=I_{c}\right)$ and oblate top $\left(I_{a}=I_{b}<I_{c}\right)$. Linear molecules are considered to be special case of symmetric top molecules for which $I_{a}=0$.

Rotational spectrum of symmetric top molecules is quite simple and easy to assign. However, for these molecules only one rotational constant can be determined experimentally. On the other hand asymmetric top molecules have complex rotational spectrum and the complexity increases with asymmetry in the molecule. In some cases the spectrum is so complicated that one needs to use special techniques like double resonance $[3,4]$ and Stark modulation to assign the energy levels corresponding to the observed transition frequencies. However, once the spectrum is assigned all the three rotational constants $A-C$ can be obtained for the molecule. Thus, one gets more experimental parameters to determine the structure for asymmetric top molecules.

In addition to accurate structure determination, rotational spectroscopy has found several other applications as well. The centrifugal distortion constants can be obtained 
by fitting experimental transitions from several rotational levels. These distortion constants depend on the various vibrational frequencies of the molecules [5]. For a simple diatomic molecule, the centrifugal distortion constant, $D_{j}$ is related to both the rotational constant and vibrational frequency and hence from the rotational spectrum one could determine the vibrational frequency [1]. For a simple diatomic molecule having vibrational frequency in the infrared region, it may appear like a more difficult and circuitous way of getting the vibrational frequency. However, the same method can be successfully used for weakly bound complexes such as Ar...Ne [6], where the stretching frequency is usually in the terahertz $(\mathrm{THz})$ region and microwave spectroscopy offers a cheaper and easier method for determining the stretching frequency and bond strength. Nuclei with nuclear spin $I \geq 1$ have non-zero quadrupole moment. This quadrupole moment interacts with molecular field gradient to give rise to nuclear hyperfine structure. However, such interaction is not possible in the case of nuclei having nuclear spin $(I) 0$ or $1 / 2$ since these nuclei are spherically symmetric and thus have no quadrupole moment. Molecules containing one or more nuclei with nuclear spin $I \geq 1$ show nuclear hyperfine structure in their rotational spectrum $[1,2,7]$. Electric field gradients within the molecules can be obtained from the nuclear hyperfine structure which gives information about electronic structure within the molecules and the nature of the chemical bonds (ionic/covalent) in the molecule. For example, in $\mathrm{HCl}$ having a covalent bond, the electric charge distribution around $\mathrm{Cl}$ is not spherical and the quadrupole coupling constant for $\mathrm{Cl}$ atom is $-67.819 \mathrm{MHz}$ [2] whereas the same in the ionic $\mathrm{KCl}$ is $<0.04 \mathrm{MHz}$ as $\mathrm{KCl}$ is ionic and $\mathrm{Cl}$ atom is $\mathrm{Cl}^{-}$, having a spherical charge distribution as in Ar.

Application of electric field causes splitting of rotational spectral lines and the $(2 J+1)$ degeneracy associated with $M_{J}$ values $\left(M_{J}=-J\right.$ to $+J$ including zero) for each $J$ level, is partially lifted. Here $J$ is the rotational quantum number of a quantum state having angular momentum $\sqrt{ }(J(J+1))$ and $M_{J}$ is the projection of angular momentum on a space fixed reference axis, $Z$, both in units of $h / 2 \pi$. This is known as the Stark effect. The extent of splitting depends on the dipole moment of the molecule and thus the measurement of this splitting leads to experimental determination of the permanent dipole moment of the molecule, in the ground state $[8,9]$.

Internal rotation within a molecule (like relative rotation of two methyl fragments in ethane molecule) can couple with the overall rotation of the molecule leading to splitting of the rotational transitions. The observed splitting depends on both the symmetry of the potential as well the barrier height for internal rotation. Thus by getting the value of splitting due to internal motions the nature of the potential and the barrier height can be determined for several molecules $[10,11]$.

In this article, experimental techniques used to observe rotational spectrum are summarized. Some details about the pulsed nozzle Fourier transform microwave (PNFTMW) spectrometer fabricated in our laboratory are given including some representative results. The limitations of this spectrometer are discussed as well and the need for a broadband spectrometer is presented. Broadband spectrometers are revolutionizing the field of microwave spectroscopy and many of the rotational spectroscopy groups in the world are building this type of spectrometer which was first developed by Pate and coworkers [12]. These spectrometers use chirped pulse technique and have a very high band width. Spectrum of a very wide range can be obtained in a single experiment with these spectrometers. Preliminary results from our attempts to produce a $1 \mathrm{GHz}$ broadband chirped pulse are also given in the last parts of this article.

\section{Experimental techniques}

Historically, many different methods have been adopted to obtain the pure rotational spectrum of molecules. Different techniques like data collection in time domain instead of frequency domain, use of cavity spectrometers and broadband spectrometers have been embraced by rotational spectroscopists. This section summarizes some important experimental techniques from initially used absorption cell spectrometers to the very recently reported broadband chirped-pulse spectrometers.

\subsection{Stark modulated spectrometers}

Initially simple absorption cell spectrometers were used to obtain rotational spectra of molecules. These spectrometers consist of three main components: a tuneable microwave source, an absorption cell which contains the gaseous molecule under investigation, typically in the pressure range of $0.01-0.1$ Torr, and a crystal detector which converts incident microwave power to an output voltage that can be measured. This spectrometer was useful for studying the rotational spectrum of stable molecules with large electric dipole moment. However, the sensitivity of this spectrometer was rather low. Due to the small energy gaps between rotational levels, the difference in population between the lower and upper levels is usually small contributing to the weak absorption signals.

Stark effect can be used to modulate the rotational spectral lines. Hughes and Wilson introduced Stark modulation into the absorption cell to increase the sensitivity of simple absorption cell spectrometers [13, 14]. These 
spectrometers are known as Stark modulated spectrometers. The modulation was accomplished by insertion of a single flat metal plate, called septum, in the centre of the rectangular waveguide absorption cell and application of a low frequency $(\sim 100 \mathrm{kHz})$ square wave voltage. Low frequency square wave voltage is used to insure that electric field remains nearly constant during the time interval when Stark components are displaced. By tuning the detector at the modulating frequency a significant increase in the sensitivity could be obtained. Furthermore if half cycle of the square wave voltage is clamped to zero volt, during the half cycle at $0 \mathrm{~V}$, un-displaced rotational lines are observed (no Stark effect), while during the other half cycle, at voltage say V, the displaced lines due to Stark effect is observed. Phase sensitive detectors are used to detect the un-displaced and displaced lines (Stark component). Since these lines are $180^{\circ}$ out of phase to each other, the Stark component appears inverted with respect to zero field (un-displaced) line when detected with phase sensitive detectors. In these spectrometers applied electric field is parallel to the electric field component of the microwave radiation so transition corresponding to $\Delta M_{J}=0$ are observed predominately $[1,2]$.

In addition to improving the sensitivity for observing rotational transitions, the Stark effect is useful in the determination of the permanent electric dipole moment of molecules as well. Both magnitude and direction of the dipole moment of a molecule could be obtained by using Stark modulated spectrometers. The accuracy of these spectrometers in determining the dipole moment was further improved by the introduction of parallel plate Stark cells [8, 15, 16]. Moreover these cells can easily be designed to get the external electric field perpendicular to the direction of the electric field of microwave radiation and thus transitions corresponding to the selection rule $\Delta M_{J}= \pm 1$ can also be measured. Only the gaseous compounds which possess permanent dipole moment and high vapour pressure could be studied using these spectrometers. By the end of 1960's almost all such compounds had been studied and interest in the field of rotational spectroscopy was declining.

\subsection{Pulsed microwave Fourier transform spectrometers}

In conventional Stark modulated spectrometers, the frequency is swept over a certain range and the signal is recorded as a function of frequency i.e. these spectrometers work in frequency domain. The time domain Fourier transform microwave spectroscopy was already introduced by Dicke and Romer in the 1950's [17-19]. Desire of better resolution and sensitivity and success of pulsed FT-NMR spectrometers inspired microwave spectroscopist to use analogous technique in microwave spectroscopy. In 1975, Ekkers and Flygare [20] came up with a new spectrometer design in which the technique of Fourier transformation was used. In this spectrometer a series of microwave pulses of certain time duration (ranging from nanoseconds to microseconds) were used. The width of frequency spectrum of a pulse depends on its time duration. For very short pulse, the width is large and whole spectrum range within this width can be measured simultaneously. Bandwidth up to $50 \mathrm{MHz}$ could be covered by a single pulse train. The power used for these pulses depends on the dipole moment of the molecule under investigation. Ekkers and Flygare showed that in a C-band wave guide absorption cell, used for molecule and microwave interaction, $11 \mathrm{~W}$ pulse power is needed to saturate a transition with transition dipole moment of $\sim 1$ Debye in a band of $50 \mathrm{MHz}$. After each pulse, the decaying time dependent polarization emission from the excited states is observed by super-heterodyne detection technique (vide infra). With this technique, resolution of spectra significantly increased due to complete absence of power and modulation broadening. The emission response after each pulse is added coherently at the detector to increase signal to noise ratio. This time domain response is Fourier transformed to give the frequency domain spectrum. The time domain spectrometers have much better sensitivity and resolution in comparison to conventional frequency domain spectrometers.

This technique was used by Dreizler and co-workers [21, 22] in several experiments. Oldani and Bauder [23] reported high resolution spectrum of $\mathrm{C}_{6} \mathrm{H}_{5} \mathrm{D}$, which has very low dipole moment, for the first time using a spectrometer based on similar technique. Furthermore they could observe deuterium nuclear quadrupole hyperfine splitting for the same compound and calculate the coupling constant from observed splitting [24]. While these improvements helped in adding a few more molecules that could be investigated by microwave spectroscopy, these were not enough to sustain interest in this area. The major disadvantage of microwave spectroscopy remained: only small molecules with sufficient vapour pressure could be investigated. Coupling of a molecular beam to microwave spectrometer enhanced the scope significantly and this is discussed next.

\subsection{Molecular beam spectrometers}

\subsubsection{Molecular beam electric resonance (MBER) spectrometer}

As Muenter [25] points out in a review, "Development of atomic and molecular beam techniques can not be separated from the development of beam spectroscopy". MBER spectroscopy is very different from all other spectroscopies in that, this technique detects molecules and not 
radiation. The sensitivity is independent of the frequency range and MBER spectroscopy can be done from $10^{5}$ to $10^{15} \mathrm{~Hz}$ [25]. In short, a molecular beam is detected by a mass detector that counts the molecules/complexes impinging on it at a given mass. An electric field intercepts the beam and deflects the molecules/complexes of a given mass only when there is a resonant frequency, leading to a dip in the observed signals. As only the molecules are detected, one could use a broadband light source for an initial search of the spectrum and then do a high resolution study once the transitions are identified.

The use of supersonic/hypersonic molecular beam leads to two important advantages. Supersonic cooling reduces the number of quantum states populated and hence the spectrum is easier to assign. In addition, it enables the formation of weakly bound complexes, which can be investigated. This enlarges the number of molecular systems that can be investigated by microwave spectroscopy, and not surprisingly, molecular beam microwave spectroscopy is still an active field. MBER played a crucial role in the history of hydrogen bonding, as it helped Klemperer and co-workers [26] to record the microwave spectrum of the hydrogen fluoride dimer. This was the first time a hydrogen bond was observed in isolation and the structure was characterized unambiguously using microwave spectroscopy. This was followed by the observation of the water dimer by Dyke and Muenter [27]. However, the MBER technique did not have enough sensitivity, certainly not comparable to the PNFTMW spectrometer, which was introduced by Balle and Flygare in 1979 [28]. A dramatic example that highlights the difference between the two techniques is the case of the benzene dimer. Molecular beam electric resonance spectrometer could not record any well-resolved signals from the benzene dimer, though the observation of weak signals was used to conclude that the dimer has dipole moment and hence it should be T-shaped [29]. Following this work, in 1992 Felker and co-workers had commented that 'benzene dimer has an apparently unresolvable rotational spectrum' [30]. The very next year Arunan and Gutowsky [31] reported the rotational spectrum of the benzene dimer using a PNFTMW spectrometer and showed that the dimer is indeed T-shaped, resolving the long controversy about its structure. The MBER technique is rarely used for microwave spectroscopy since the late $1980 \mathrm{~s}$ and almost all the microwave laboratories started using the PNFTMW spectrometer, which is discussed next.

\subsubsection{PNFTMW spectrometer}

A major thrust to microwave spectroscopy was given by Balle and Flygare, who coupled a supersonic nozzle source to Fourier transform microwave spectrometer designing the first PNFTMW spectrometer [28]. This spectrometer consists of mainly (1) Fabry-Perot cavity. (2) Supersonic nozzle to prepare a molecular beam. (3) RF and MW electrical circuit to send the microwave radiations inside the cavity and to detect the emitted signal.

The Fabry-Perot cavity is the backbone for these spectrometers. In the original design the cavity was formed by two parallel, concave and highly polished aluminium mirrors with a diameter of $36 \mathrm{~cm}$ and a radius of curvature (R) $84 \mathrm{~cm}$. The two mirrors were separated by $50-70 \mathrm{~cm}$ (D) apart from each other. A typical Fabry-Perot cavity is shown in Fig. 1. Mirror radius $(a)$ and radius of curvature $(R)$ determine the lower frequency limit for the cavity, at which Fresnel's number is unit as given below:

$\frac{a^{2}}{R \lambda}=1$

where $\lambda$ is the wavelength.

In the original design, this condition sets the frequency lower limit to $7,770 \mathrm{MHz}$. A wave-guide was used to couple the microwave radiation inside the Fabry-Perot cavity and hence the experiments were done in the octave bands $\mathrm{X}(8-12 \mathrm{GHz})$ and $\mathrm{Ku} 12-18(\mathrm{GHz})$ only i.e. frequency range for this spectrometer was $8-18 \mathrm{GHz}$ [25]. The mirrors inside the cavity were mounted in a way that one of the mirrors can be moved and the distance between the two mirrors can be varied in steps of microns. The cavity can support an infinite number of standing wave patterns called modes. These are designated as $\operatorname{TEM}_{m n q}$ modes where $m, n$ and $q$ denote the number of nodes in the three orthogonal directions, $q$ along the cavity axis. The dominant modes for the Fabry-Perot cavity are of the type $\mathrm{TEM}_{00 \mathrm{q}}$. Due to very high quality factor, Q-factor $\left(\sim 10^{4}\right.$ or more), these spectrometers have very low bandwidth, typically of the order of $1 \mathrm{MHz}$. This also means that the microwave power needed in these experiments is low, typically a few milliwatts. The nozzle was situated on top of the cavity and the molecular beam travelled perpendicular to the microwave radiations. If the molecular system under investigation has an allowed transition within

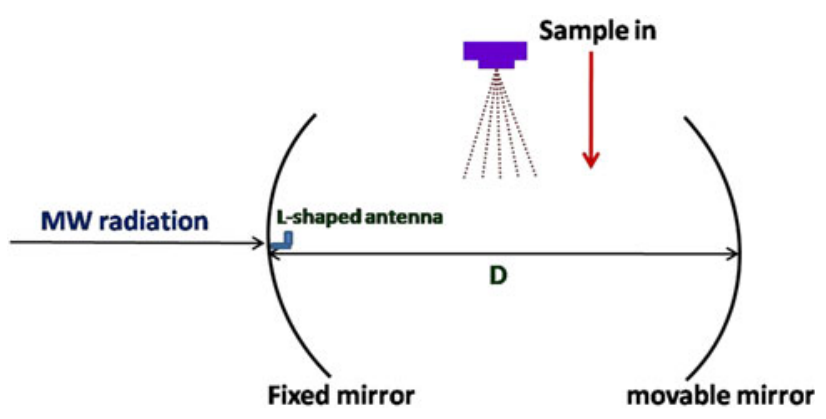

Fig. 1 A typical Fabry-Perot cavity used in pulsed nozzle Fourier transform microwave spectrometer 
the bandwidth of the cavity $\Delta v$ centred at $v$ (master oscillator, MO) it can be excited. The emission from the excited sample at $v \pm \Delta v$ is detected by double super-heterodyne detection technique. Basically, the signal at the frequency $v \pm \Delta v$, which could be several $\mathrm{GHz}$, is brought down to $30 \pm \Delta v \mathrm{MHz}$ by mixing with $v-30$ (local oscillator, LO) in one stage and brought down to $\Delta v$ (which is within $1 \mathrm{MHz}$ ) by mixing it with a $30 \mathrm{MHz}$ signal in the next stage. Phase matching for these two oscillators was the most tedious job while operating these spectrometers. The time domain response collected by these spectrometers was further Fourier transformed to give frequency domain signal.

In our lab, we use a spectrometer based on BalleFlygare design with some modification, which are discussed in next section. Details of our spectrometer have been reported earlier [32]. Since then we have made some minor changes in our spectrometer. Current schematic of the spectrometer is shown in Fig. 2. As mentioned above, the cavity is tuned by moving one of the mirrors while monitoring the reflected signal which shows a dip when the cavity is tuned: see Fig. 3a, b for the reflected signals when the cavity is not tuned and tuned, respectively.

\subsubsection{Advancements in PNFTMW design}

After its invention by Balle and Flygare, the PNFTMW spectrometer has seen much advancement. The first changes were made by Suenram et al. [33] and Kruger et al. [34]. Instead of using $2 \mathrm{~mW}$ oscillators they used one $\mathrm{mw}$ source as local oscillator and one single side band mixer (SSBM). The master oscillator was produced by the mixing of an RF frequency (typically $30 \mathrm{MHz}$ ) with the LO frequency in the SSBM. This arrangement eliminated the need of the tedious phase matching required in the original design.

Second major advancement was in the nozzle position. Grabow and Stahl [35] moved the nozzle from top of the cavity to behind the fixed mirror. In this arrangement the molecular beam and microwave radiations are coaxial to each other. This increases the interaction time between molecular beam and radiation, which in turn reduces the line width to a few $\mathrm{KHz}$. This enables observation of very small hyperfine splitting also.

The frequency range accessible with the original BalleFlygare spectrometer was $8-18 \mathrm{GHz}$. This range has been extended on both side and the spectrometers operating from 1 to $40 \mathrm{GHz}$ are available now [36, 37]. The most significant contributions towards low frequency region $(<2 \mathrm{GHz})$ are due to Grabow's and Cooke's groups. Grabow and coworkers [36] presented a different type of cylindrical resonator in which $\mathrm{TE}_{01}$ modes were used in contrast to TEM $_{00 \mathrm{q}}$ modes used in Fabry-Perot spectrometers. This spectrometer works well in low frequency range and they could get signal as low as $1325.17 \mathrm{MHz}$ for 1-azabicyclo[2]-octane... $\mathrm{H}_{2} \mathrm{~S}$ complex [36]. On the other hand the spectrometer constructed by Cooke and co-workers [38] uses the same TEM $\mathrm{TOq}_{\mathrm{q}}$ mode as used in Fabry-Perot design. This spectrometer uses mirrors which have equal value of radius and radius of curvature $(a=R=20 \mathrm{~cm})$. The lowest frequency transition reported by Cooke and coworkers [39] using this spectrometer is $805.1594 \mathrm{MHz}$ for pivaloyl chloride, ${ }^{37} \mathrm{Cl}$ isotopologue.

There have been several ingenious modifications to the nozzle design to expand the range of systems that could be investigated. High temperature nozzles are used to study less volatile species and pyrolysis products. Moreover rotational spectra in vibrationally excited states can also be observed by the use of hot nozzles. Shea and Campbell [40] reported the first application of high temperature nozzle in PN-FTMW for the study of $\mathrm{Hg}$... $\mathrm{HCl}$ complex. Fast mixing nozzles are used to form complexes between compounds which react very fast when mixed. This type of nozzle was first reported by Emilsson et al. [41] to study $\mathrm{NH}_{3} \ldots \mathrm{HCN} \ldots \mathrm{HF}$ and $\mathrm{CO} \ldots \mathrm{HCN} \ldots \mathrm{HF}$ trimers. Pulsed discharge nozzles, in which an electric discharge is used right after the nozzle, are used to study ions, radicals and their complexes. Several radical species like $\mathrm{C}_{3} \mathrm{~S}, \mathrm{C}_{2} \mathrm{~N}$, $\mathrm{NCCS}, \mathrm{HC}_{3} \mathrm{~S}$ etc. have been studied using these nozzles $[42,43]$. Studies on rare gas-ion/radical complexes such as $\mathrm{Ar}-\mathrm{SH}$ [44], $\mathrm{Ar}-\mathrm{NH}_{2}{ }^{+}$and $\mathrm{Kr}-\mathrm{NH}_{2}{ }^{+}$[45] have also been reported. Laser ablation technique has also been added to the nozzle source. Using this technique new species can be generated and studied. This technique facilitates the study of refractive materials, reactive and low volatile materials. First application of this technique was done by the NIST group to study $\mathrm{SiC}_{2}$ [46]. Since then many metal oxides and solid organic compounds have been studied using this technique [47-50].

In our laboratory, the PNFTMW spectrometer has been used to investigate several weakly bound complexes containing $\mathrm{H}_{2} \mathrm{O} / \mathrm{H}_{2} \mathrm{~S}$ [51]. The complexes investigated so far include $\mathrm{C}_{2} \mathrm{H}_{4} \ldots \mathrm{H}_{2} \mathrm{~S}$ [52], $\mathrm{Ar}_{2} \ldots \mathrm{H}_{2} \mathrm{~S}$ [53], $\mathrm{Ar} \ldots\left(\mathrm{H}_{2} \mathrm{~S}\right)_{2}$ [54], Ar... $\mathrm{H}_{2} \mathrm{O} \ldots \mathrm{H}_{2} \mathrm{~S}$ [55], phenylacetylene $\ldots \mathrm{H}_{2} \mathrm{O} / \mathrm{H}_{2} \mathrm{~S}[56,57]$, $\mathrm{CH}_{4} \ldots \mathrm{H}_{2} \mathrm{~S}$ [58] and $\mathrm{C}_{6} \mathrm{H}_{6} \ldots \mathrm{C}_{2} \mathrm{H}_{4}$ [58]. Our interest in complexes involving $\mathrm{H}_{2} \mathrm{O}$ and $\mathrm{H}_{2} \mathrm{~S}$ is the result of our desire to understand the physical forces involved hydrogen bonding and van der Waals interactions. The importance of hydrogen bonding should be well known to chemists and biologists as it controls the properties of the molecules of life, namely $\mathrm{H}_{2} \mathrm{O}$ and DNA. The fact that $\mathrm{H}_{2} \mathrm{O}$ is a liquid and $\mathrm{H}_{2} \mathrm{~S}$ is a gas at room temperature led to the historical view that $\mathrm{OH}$ groups can form hydrogen bonds and $\mathrm{SH}$ groups cannot. This was further strengthened by the fact that $\mathrm{H}_{2} \mathrm{O}$ has only four neighbours when it freezes and $\mathrm{H}_{2} \mathrm{~S}$ has 12 i.e. each $\mathrm{H}_{2} \mathrm{O}$ molecule is hydrogen bonded to four 


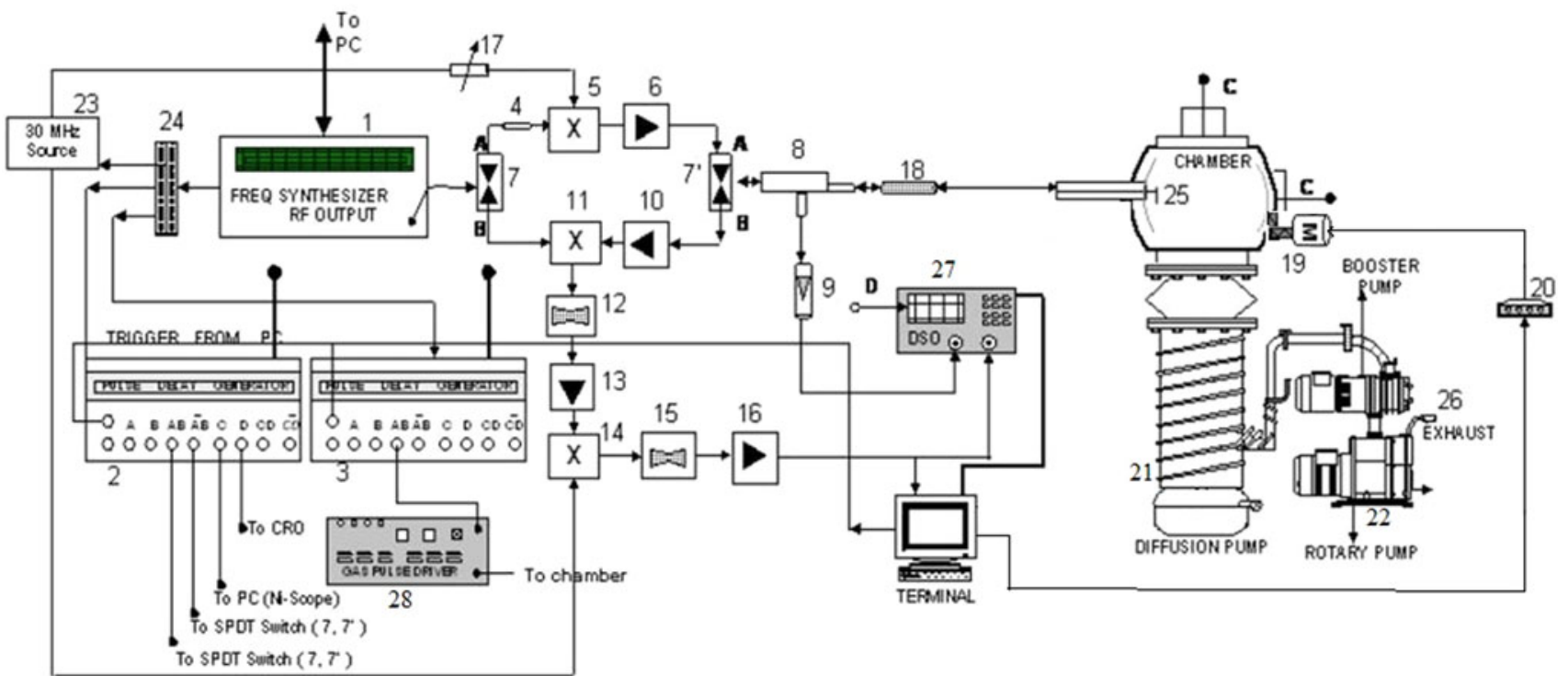

Fig. 2 Schematic diagram of the PNFTMW spectrometer at the Indian Institute of Science. 1 frequency synthesizer (HewlettPackard, HP83630L), 2 and 3 delay generator (SRS DG535), 4 microwave attenuator (HP, 8493C, 3dB), 5 SSB mixer (Miteq, SMO-226LC1A), 6 medium power amplifier (Miteq, JS3-02002600-5-7A), 7 MW SPDT switch (Sierra Microwave, 0.5-26.5 SFD0526-000), 8 direction coupler (Narda, 1.7-26.5-4227-16), 9 diode detector (Narda, 0.01-26.5-4507), 10 low noise amplifier (Miteq, JS4-02002600-35P), 11 image rejection mixer (Miteq, IRO-0226LC1A), 12 band pass filter (Mini Circuits, BBP-30); 13 and 16 RF amplifier (Mini Circuits, ZFL-500LN), 14 RF mixer (Mini Circuits, ZAD-1), 15 low pass filter (Mini Circuits, BLP-5), 17 attenuator (Mini Circuits, ZAFT-51020), 18 blocking capacitor (HP, 11742A), 19 stepper motor; 20 motor driver, 21 diffusion pump and 22 rotary pump (Vacuum Techniques, Bangalore, India), $2330 \mathrm{MHz}$ function generator (Stanford Research System, DS345), 24 distributionamplifier (Stanford Research System, FS710), 25 antenna, 26 exhaust line, 27 digital storage oscilloscope, and 28 pulse valve driver
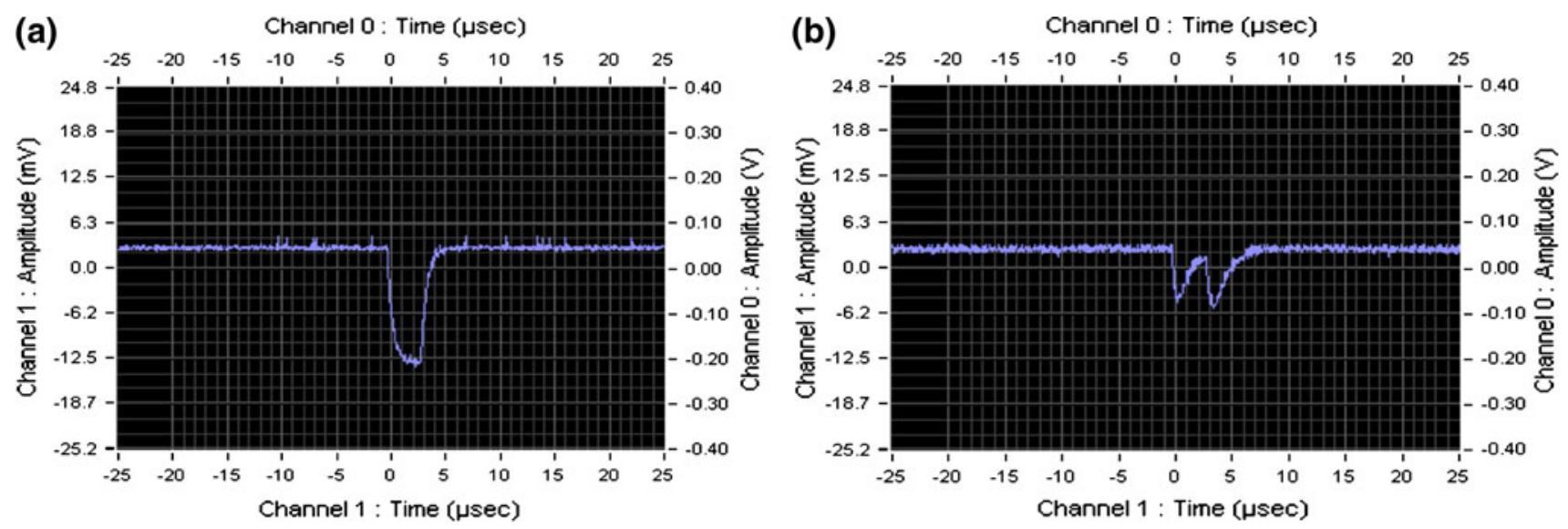

Fig. 3 Oscilloscope display a when cavity is not tuned $\mathbf{b}$ when cavity is tuned

other $\mathrm{H}_{2} \mathrm{O}$ molecules as donor through the two $\mathrm{OH}$ groups and acceptors through the two lone-pairs in the $\mathrm{O}$ atom. In $\mathrm{H}_{2} \mathrm{~S}$, there is no such directionality in interaction and each molecule interacts with 12 others in an isotropic fashion and in essence, the $\mathrm{H}_{2} \mathrm{~S}$ is spherical. This led the chemists and biologists to believe that $\mathrm{H}_{2} \mathrm{O}$ has hydrogen bonding which originates from a distinct physical force different from $\mathrm{H}_{2} \mathrm{~S}$ which is bound by 'van der Waals forces'. Our experimental results in a supersonic beam resulted in a different picture. The $\mathrm{H}_{2} \mathrm{~S}$ dimer had a structure very similar to the well known $\mathrm{H}_{2} \mathrm{O}$ dimer structure [55].
Moreover, $\mathrm{C}_{2} \mathrm{H}_{4} \ldots \mathrm{H}_{2} \mathrm{O}$ [59] and $\mathrm{C}_{2} \mathrm{H}_{4} \ldots \mathrm{H}_{2} \mathrm{~S}$ have similar geometry that can be characterized as $\mathrm{O}-\mathrm{H} \ldots \pi$ and $\mathrm{S}-\mathrm{H} . . . \pi$ hydrogen bonding interactions. A detailed analysis of the intermolecular potential revealed that the difference between the condensed matter behaviour and that found in the supersonic beam is just the thermal energy along torsional coordinates that break the hydrogen bonds [60]. This led us to propose a dynamic criterion for a hydrogen bond [60] which is universally applicable. Moreover, structural results from microwave spectroscopy of hydrogen bonded complexes helped us to define 
hydrogen bond radii various donors and acceptors [61, 62]. Finally, these results led us to propose a modern definition for hydrogen bonding, in an effort initiated through IUPAC [63]. Very recently, the $\mathrm{C}$ rotational constant of ${ }^{13} \mathrm{CC}_{5} \mathrm{H}_{6}$ having nearly zero dipole moment was determined by measuring the transitions of the ${ }^{13} \mathrm{CC}_{5} \mathrm{H}_{6} \ldots$ Ar complex, in which Ar could be considered an electrophore, inducing a dipole moment along the $C$ axis of ${ }^{13} \mathrm{CC}_{5} \mathrm{H}_{6}$. [64]. For a detailed review on PNFTMW spectrometer, its advancements and applications reader is referred to Ref. [65].

\subsection{Limitations of PNFTMW and the need of broadband spectrometer}

The PNFTMW spectrometers provide very high resolution and sensitivity, simultaneously and have enjoyed wide range of application as mentioned above. However, these cavity spectrometers have two very serious limitations: (i) very low bandwidth which makes searching for unknown transitions long and tedius and (ii) low frequency cut (see Eq. 1) off near about $4 \mathrm{GHz}$ for most of the spectrometers. Though it is now possible to do gas phase spectroscopy on larger molecules and complexes, the moments of inertia for these are much larger and the rotational constants are smaller. It would be desirable to have a spectrometer with similar sensitivity and resolution down to $1 \mathrm{GHz}$ or less.

Today microwave spectroscopists are trying to target heavier molecules and larger complexes. With increase in molecular mass, the rotational constants decrease and most of the low $J$ transitions which are usually the most informative, lie in the low frequency region. As an example, consider a T-shaped structure of phenylacetylene-benzene complex, in which acetylenic hydrogen is interacting with the $\pi$ cloud of benzene. The rotational constants for this structure are calculated to be $A=1907.45 \mathrm{MHz}$, $B=189.17 \mathrm{MHz}$ and $C=183.13 \mathrm{MHz}$ at B3LYP/6$311++\mathrm{G}(2 \mathrm{~d}, 2 \mathrm{p})$ level. The $O_{0,0}-1_{0,1}$ transition for this complex is expected at $372.3 \mathrm{MHz}$. All the frequencies corresponding to $J=2-3$ transitions for this complex range from 725 to $1,130 \mathrm{MHz}$. These transitions are not accessible with the typical Fabry-Perot PN-FTMW spectrometers. More interestingly, one can consider the benzene dimer which is also expected to have a minimum having both benzene molecules parallel to each other, slightly displaced [66]. As this would not have a dipole moment, microwave spectroscopy cannot be used to investigate such a structure. If $\mathrm{C}_{6} \mathrm{H}_{6} \ldots \mathrm{C}_{6} \mathrm{H}_{6} \ldots$ Ar trimer could be formed in which the two benzene molecules have parallel-displaced geometry, microwave spectroscopy can given unambiguous evidence for the same. However, the rotational transitions for such a complex are also expected below $1 \mathrm{GHz}$.
These limitations show the need for a new spectrometer which can work in low frequency range (below $2 \mathrm{GHz}$ ) and can perform data collection over a broad frequency range in a short time. Pate and co-workers came up with a novel design, which has given the most significant fillip to microwave spectroscopy after the development of PNFTMW spectrometer by Balle and Flygare and the details are given next.

\subsection{Broadband spectrometer}

Pate and co-workers [67] introduced the use of chirped pulse in microwave spectroscopy. The PNFTMW spectrometers typically use a $1 \mu \mathrm{s}$ pulse of monochromatic continuous wave microwave radiation, say at $10 \mathrm{GHz}$. This pulse results in a narrow bandwidth of about $1 \mathrm{MHz}$ centred at $10 \mathrm{GHz}$, limiting the bandwidth observed in every experiment. In a chirped pulse of the same duration, $1 \mu \mathrm{s}$, frequency is swept between a high and low frequency and the difference could be even $11 \mathrm{GHz}$ i.e. in a chirped pulse the frequency is linearly changing with time covering a much broader range of frequencies.

Pate and co-workers [67] presented two designs for the new broadband spectrometer. In one design, a chirp pulse of frequency from 1537.5 to $162.5 \mathrm{MHz}$ with a linear frequency sweep of $1.375 \mathrm{GHz}$ is generated by a 4.2 Gsample/s arbitrary waveform generator (AWG). The time duration of the chirp is $1 \mu \mathrm{s}$. After several multiplication and modulations processes the band-width of the chirp is increased by a factor of 8 and it is up-converted to a chirp spanning from 7.5 to $18.5 \mathrm{GHz}$. This MW chirp after amplification by a travelling wave tube amplifier, is sent into a vacuum chamber by a broadband horn antenna. Molecular sample interacts with this chirp in the vacuum chamber and the broadband free induction decay (FID) is collected by another horn antenna. This broadband FID is down converted to the $0.5-11.5 \mathrm{GHz}$ band and digitized by a 40 Gsample/s, $12 \mathrm{GHz}$ band-width digital oscilloscope. In the second design, a chirp of $12 \mathrm{GHz}$ band-width is directly generated by a 20 Gsample/s AWG and is up converted in a single step by an ultra broadband mixer. The molecular emission is directly detected (no down conversion) by a $50 \mathrm{Gsample/s} \mathrm{digital} \mathrm{oscilloscope} \mathrm{with} 18 \mathrm{GHz}$ bandwidth. In both the designs, the FIDs are averaged in time domain to increase $\mathrm{S} / \mathrm{N}$ ratio and then fast Fourier transformed to give frequency domain signal. Thus a spectrum covering up to $12 \mathrm{GHz}$ range can be collected at a time! Since no mirrors are used in these spectrometers and the frequency range explored at a time with these spectrometers is almost $10^{4}$ orders of magnitude more than PNFTMW spectrometers, the microwave power requirement is very high in these spectrometers. Pate et al. report that the power required for polarizing molecules with 
dipole moment more than $0.7 \mathrm{D}$ with $1 \mu \mathrm{s}, 11 \mathrm{GHz}$ chirp pulse is $2 \mathrm{~kW}$, which can be compared to a few $\mathrm{mW}$ power used in PNFTMW spectrometers. However, investigating the microwave spectrum of a molecule/complex with a PNFTMW spectrometer can be compared to looking through a key-hole to identify what is inside a room. Pate's new design has opened the door for microwave spectrum of molecules/complexes.

\section{Initial attempts to produce a chirped pulse of $1 \mathrm{GHz}$}

We are planning to build a spectrometer which works in $1-12 \mathrm{GHz}$ frequency range and uses the chirp pulse technique. Considering the reliability of ab initio calculations in geometry optimization and thus in predicting rotational constants, we focused on generation of a chirp pulse of $1 \mathrm{GHz}$ bandwidth and $1 \mu$ s time duration. We used Agilent N8241A AWG and E8267D PSG vector signal generator (VSG) for the chirp pulse generation.

The N8241A is a dual channel AWG which has two options: options 125 and 062. Under option 125 each channel can simultaneously deliver 1.25 Gsample/s with 15 bits of vertical resolution, and $500 \mathrm{MHz}$ of instantaneous analog bandwidth while under option 062 each channel delivers $250 \mathrm{MHz}$ of bandwidth at sampling rate $625 \mathrm{MS} / \mathrm{s}$ with 15 bits of vertical resolution. The E8267D
PSG VSG can deliver frequency ranging from $250 \mathrm{KHz}$ to $44 \mathrm{GHz}$ with $0.001 \mathrm{~Hz}$ resolution. It has a built in wideband I/Q modulator that delivers up to $2 \mathrm{GHz}$ RF modulation bandwidth and thus can be used as a I/Q upconvertor also. Using E8267D in compliment to N8241A AWG as I/Q up-convertor one can get modulation bandwidth of $1 \mathrm{GHz}$ (at $1.25 \mathrm{Gsample} / \mathrm{s}$ ) or $500 \mathrm{MHz}$ (at $625 \mathrm{Msample} / \mathrm{s}$ ) at MW frequencies. We used option 125 of AWG to generate a chirp of $1 \mathrm{GHz}$ after up-conversion by VSG. The setup for the chirp generation is shown in Fig. 4.

The start, end and center frequencies and the time duration of the chirp can be set by the VSG. As a very first test, the chirp output (with $5 \mathrm{GHz}$ center frequency, $1 \mathrm{GHz}$ span and $1 \mu$ s time duration) from VSG was directly fed to the Spectrum analyzer (Agilent, E4407B ESA-E Spectrum Analyzer $100 \mathrm{~Hz}-26.5 \mathrm{GHz}$ ) which displays the generated chirp. After this direct measurement, two wideband horn antennae $(2-18 \mathrm{GHz})$ were introduced between the VSG and the spectrum analyzer. One was connected to VSG and was used as transmitter of chirp pulse and the other was connected to the spectrum analyzer and was used as receiver of chirp pulse. The two horn antennae were placed close to each other and the chirp was recorded in spectrum analyzer. In Fig. 5, the frequency spectra shown in blue colour (top) is the result of measurement when VSG output was directly connected to the spectrum analyzer while

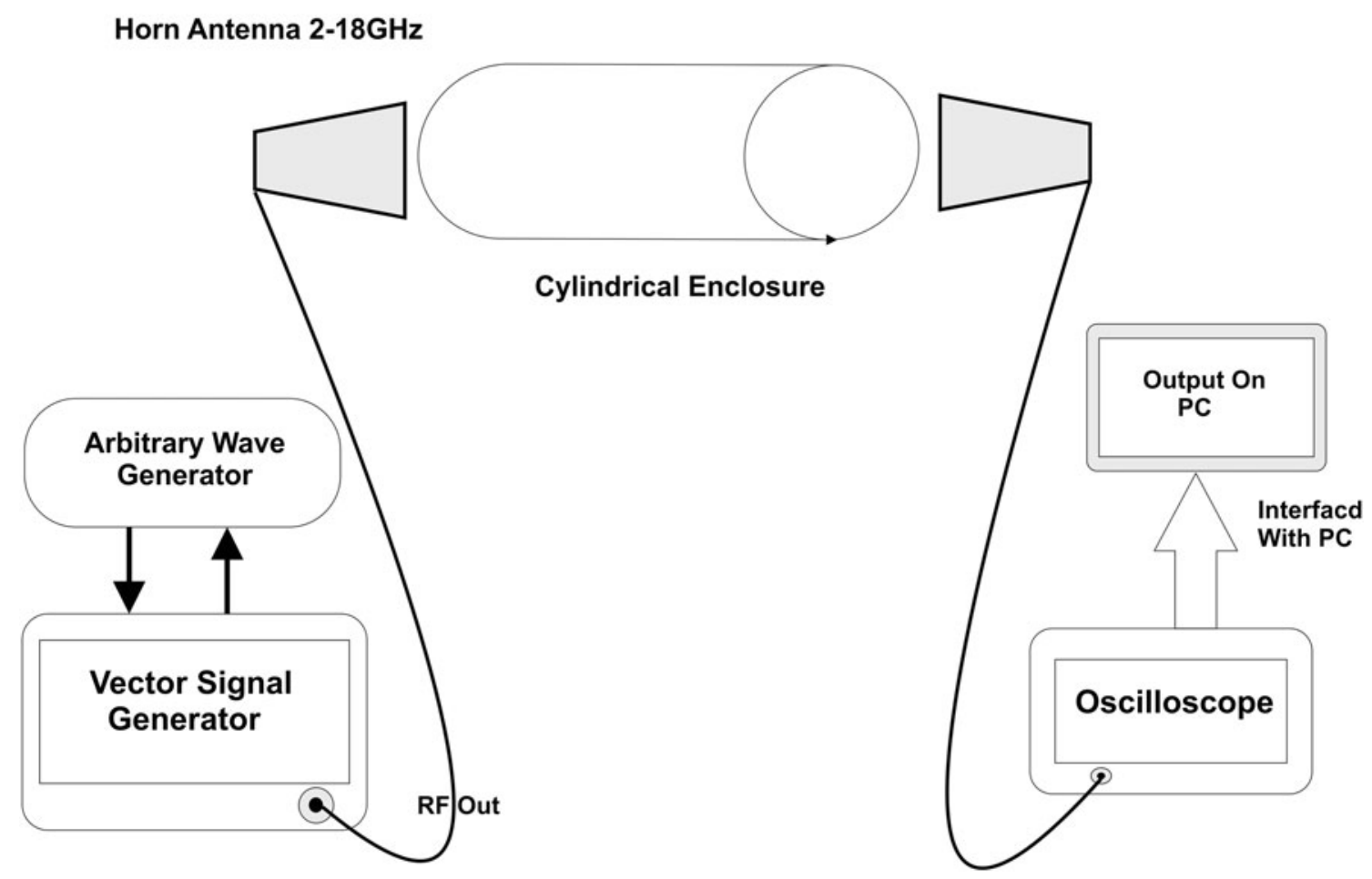

Fig. 4 Setup for chirp generation and measurements 


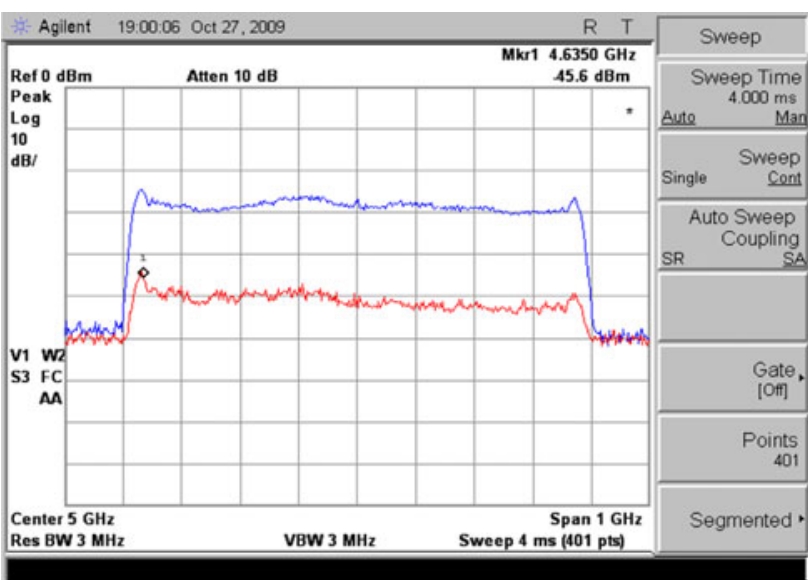

Fig. 5 Chirp output measured by spectrum analyzer, for description see text

signal shown in red colour (bottom) is obtained when horn antennae were used as transmitter and receiver. It is clear that the spectra are similar in both the cases but the amplitude decreases on using horn antennae as expected.

To measure the linearity of the chirp and phase variation with time we fed the chirp output to a wide band oscilloscope (DSA 1304A infiniium high performance oscilloscope with $13 \mathrm{GHz}$ bandwidth) and interfaced the oscilloscope with a PC as shown in Fig. 4. The PC runs a software called 'Vector signal analyzer' developed by Agilent, which shows the frequency and time domain chirp signal, frequency and phase variation of chirp with time. Figure 6 shows the result of direct measurement (without using horn antennae) using this software. In this figure, the user interface of VSA software is shown. The two graphs in first column of the figure show frequency and time domain chirp pulse respectively while in the second column, the top graph shows variation of chirp frequency with time and the bottom graph shows variation of phase of the chirp with

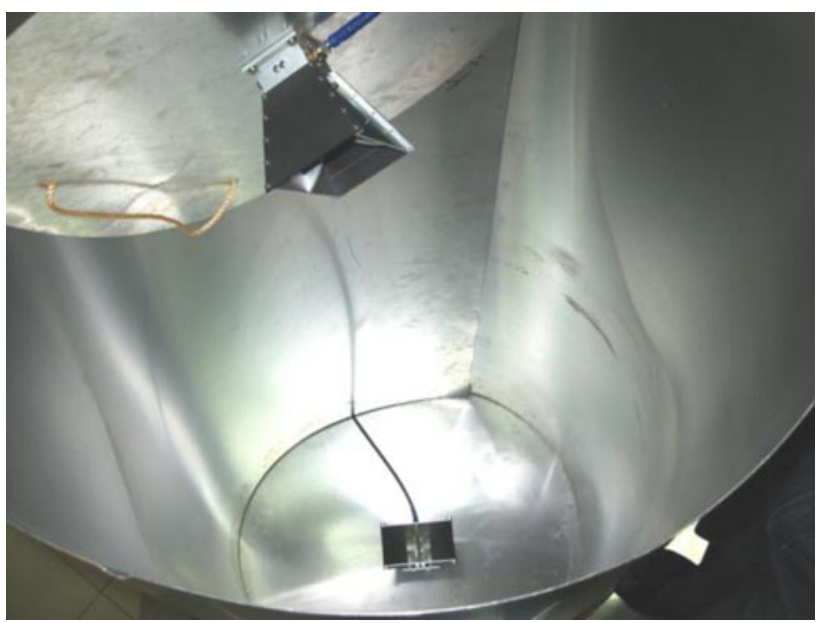

Fig. 7 The temporary arrangement for testing chirp performance inside the metal cavity

time for $1 \mu \mathrm{s}$ time duration. The chirp range is same as before $(4.5-5.5 \mathrm{GHz})$. It is clear from this measurement that the frequency of generated chirp varies linearly with time.

The horn antennae have to be introduced into a vacuum chamber in our experiment. Hence, it was decided to test how the reflections from metal chamber would affect the chirp. Moreover, the horn antennae will be separated by a finite distance in the spectrometer, and it would be useful to see the effect of varying distances between the antennae on the chirp. For these tests, a temporary arrangement was made using an aluminium sheet, folded to give the shape of a cylindrical cavity. The two horn antennae were mounted on two circular aluminium sheets. The diameter of one circular sheet was taken more than that of cylinder while diameter of other circular sheet was taken slightly less than cylinder. One horn antenna (mounted on bigger circular sheet) was kept static on one side of the cylinder while the
Fig. 6 Result of direct measurement of chirp obtained using VSA software
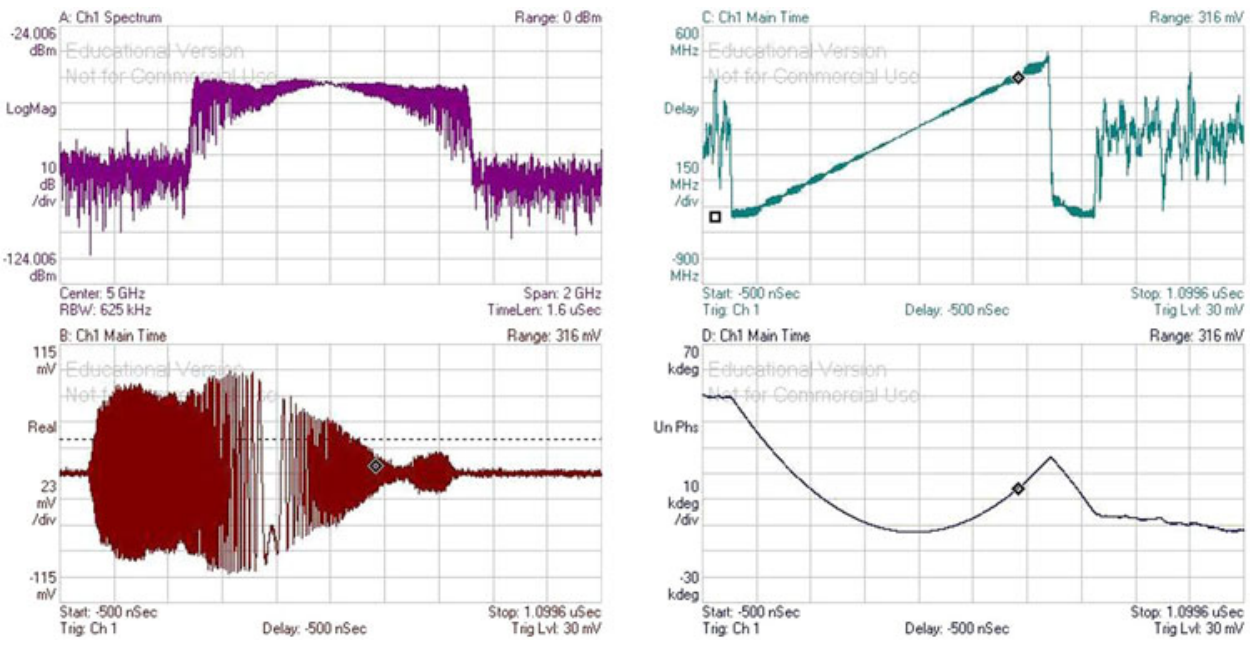
Fig. 8 Effect of putting horn antennae inside the metal cavity at different distances. a Distance between antennae $=50 \mathrm{~cm}, \mathbf{b}$ distance between antennae $=100 \mathrm{~cm}$ (a)
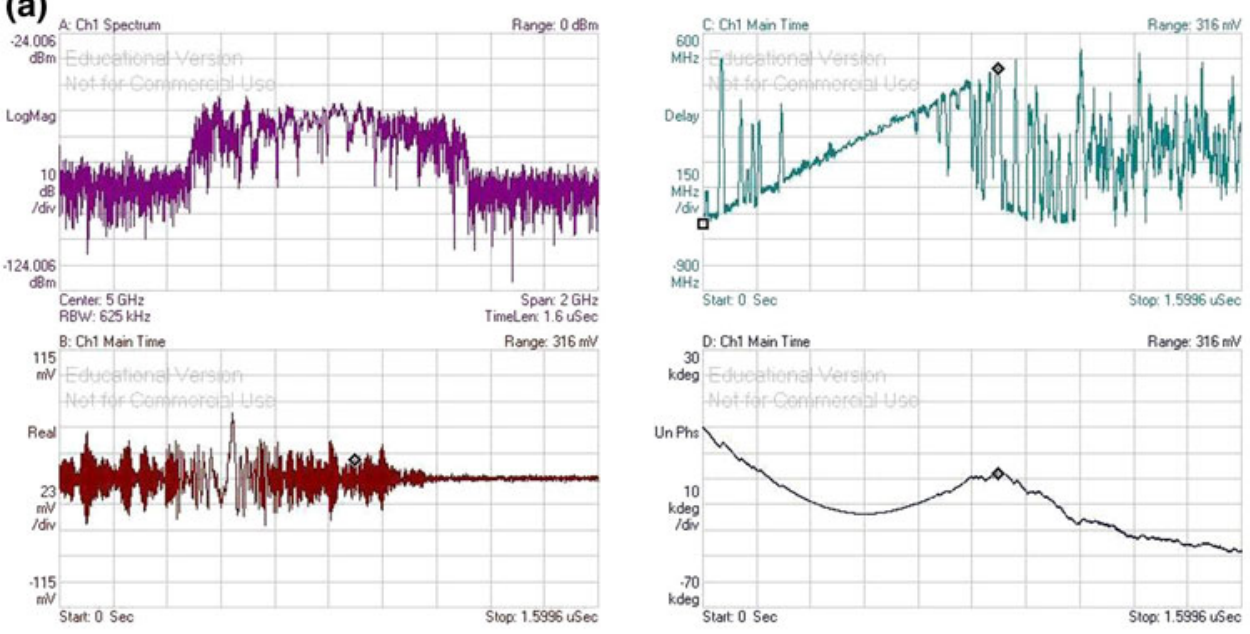

(b)

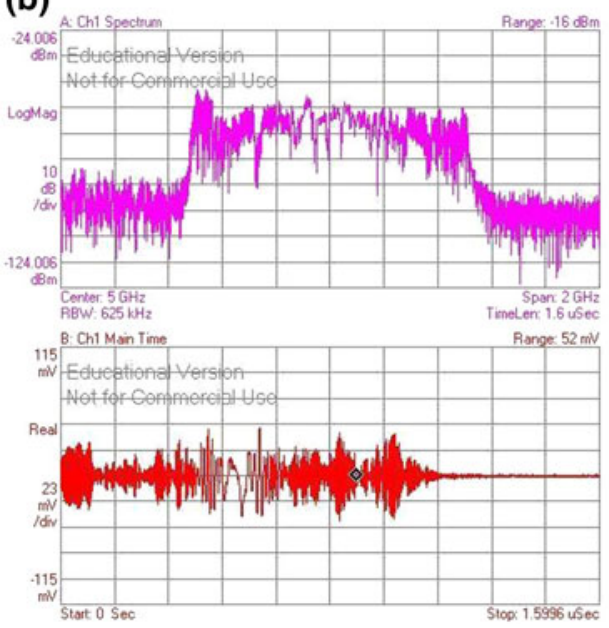

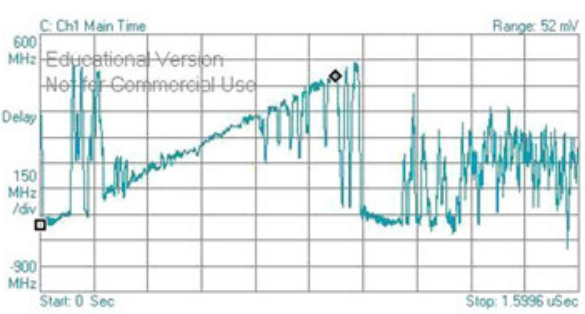

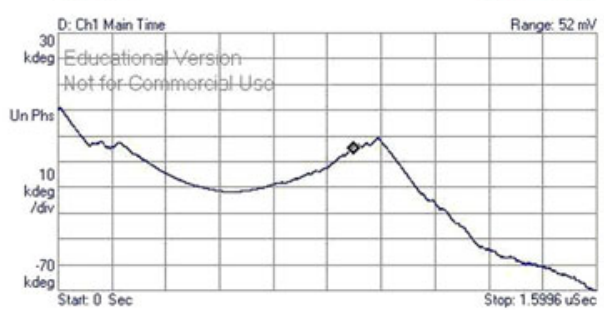

other one was moved inside the cylindrical cavity to vary the distance between two antennae. This arrangement is shown in Fig. 7. By moving the antenna inside the cylindrical cavity, we did measurements at several distances. Figure $8 \mathrm{a}, \mathrm{b}$ show the measurement results at 50 and $100 \mathrm{~cm}$ distances, respectively, between the two antennae. The chirp shows minor distortion possibly due to reflections of radiations from cylindrical cavity. However, the most important result is that at both the distances 50 and $100 \mathrm{~cm}$, the linearity of the chirp with time is maintained inside the cavity. Moreover, for both the distances the phase variation is same as for the direct measurement result shown in Fig. 5.

\section{Conclusions}

A brief review on techniques used to obtain rotational spectra of molecules and complexes has been presented. The pulsed nozzle Fourier transform microwave spectrometer, fabricated in our laboratory has been discussed at length including some important results. These have led us to define hydrogen bond radii for the various donors and acceptors and also to propose a modern definition for hydrogen bonding through IUPAC. However, the PNFTMW spectrometer has some limitations in terms of the narrow bandwidth and the lower frequency cut off. Both of these can be overcome by the new chirped pulse Fourier transform microwave (CPFTMW) spectrometer originally designed by Pate and co-workers. Currently, our laboratory is planning to construct a CPFTMW spectrometer and some preliminary experimental results have been reported here. The construction of this new spectrometer will reduce the time taken for data collection and it should also enable us to study large molecules and complexes like phenylacetylene...benzene and $\operatorname{Ar} \ldots\left(\mathrm{C}_{6} \mathrm{H}_{6}\right)_{2}$.

Acknowledgments EA acknowledges funding from Department of Science and Technology, Council for Scientific and Industrial Research, Indian Institute of Science, International Union of Pure and 
Applied Chemistry and the Indo-French Centre for Promotion of Advanced Research. DM acknowledges CSIR for the Junior Research Fellowship.

\section{References}

[1] C H Townes and A L Schawlow Microwave Spectroscopy (New York: McGraw Hill) (1955)

[2] W Gordy and R L Cooke Microwave Molecular Spectra (New York: Wiley) (1984)

[3] D Christen, L H Coudert, R D Suenram and F J Lovas J. Mol. Spectrosc. 17257 (1995)

[4] D Christen, L H Couder, J A Larsson and D Cremer J. Mol. Spectrosc. 205185 (2001)

[5] Th Brupbacher, J Makarewicz and A Bauder J. Chem. Phys. 101 9736 (1994)

[6] J U Grabow et al. J. Chem. Phys. 1021181 (1995)

[7] H A Fry and S G Kukolich J. Chem. Phys. 764387 (1982)

[8] S A Marshall and J Weber Phys. Rev. 1051502 (1957)

[9] O Desyatnyk, L Pszczolkowski, S Thorwirth, T M Krygowski and Z Kisiel Phys. Chem. Chem. Phys. 71708 (2005)

[10] E Tannenbaum, R J Myers and W D Gwinn J. Chem. Phys. 25 42 (1956)

[11] J Nakagawa, M Imachi and M Hayashi J. Mol. Struct. 112201 (1984)

[12] G G Brown, B C Dian, K O Douglass, S M Geyer and B H Pate J. Mol. Spectrosc. 238200 (2006)

[13] R H Hughes and E B Wilson Phys. Rev. 71562 (1947)

[14] K B McAfee, R H Hughes and E B Wilson Rev. Sci. Instrum. 20 821 (1949)

[15] B N Bhattacharya, W Gordy and O Fujii Bull. Am. Phys. Soc. 2 213 (1957)

[16] B N Bhattacharya and W Gordy Phys. Rev. 119144 (1960)

[17] R H Dicke Phys. Rev. 9399 (1954)

[18] R H Dicke and R H Romer Rev. Sci. Instrum. 26915 (1955)

[19] R H Romer and R H Dicke Phys. Rev. 99532 (1955)

[20] J Ekkers and W H Flygare Rev. Sci. Instrum. 47448 (1976)

[21] G Bestmann, H Dreizler, E Fliege and W Stahl J. Mol. Struct. 97 $215(1983)$

[22] G Bestmann, H Dreizler, H Mader and U Andresen Z. Naturforsch. 35392 (1980)

[23] M Oldani and A Bauder Chem. Phys. Lett. 1087 (1984)

[24] M Oldani, T -K Ha and A Bauder Chem. Phys. Lett. 115317 (1985)

[25] J S Muenter Atomic and Molecular Beam Methods, vol. II (G. Scoles, D Leine and U Valbusa Eds) (New York: Oxford University Press) p. 15 (1992)

[26] T R Dyke, B J Howard and W Klemperer J. Chem. Phys. 56 2442 (1972)

[27] T R Dyke and J S Muenter J. Chem. Phys. 602929 (1974)

[28] T J Balle and W H Flygare Rev. Sci. Instrum. 5233 (1981)

[29] K C Janda, J C Hemminger, J S Winn, S E Novick, S J Harris and W Klemperer J. Chem. Phys. 631419 (1975)

[30] B F Henson, G V Hartland, V A Venturo and P Felker J. Chem. Phys. 972189 (1992)

[31] E Arunan and H S Gutowsky J. Chem. Phys. 984294 (1993)

[32] E Arunan, A P Tiwari, P K Mandal and P C Mathias Curr. Sci. $82533(2002)$
[33] R D Suenram, F J Lovas, G T Fraser, J Z Gillies, C W Gillies and M Onida J. Mol. Spectrosc. 137127 (1989)

[34] M Kruger and H Dreizler Z. Naturforsch. 45a 724 (1990)

[35] J U Grabow and W Stahl Z. Naturforsch. 45a 1043 (1990)

[36] V Storm, H Dreizler, D Consalvo, J U Grabow and I Merke Rev. Sci. Instrum. 672714 (1996)

[37] I Merke, W Stahl and H Dreizler Z. Naturforsch. 49a 490 (1994)

[38] K C Etchison, C T Dewberry, K E Kerr, D W Shoup and S A Cooke J. Mol. Spectrosc. 24239 (2007)

[39] G S Grubbs II, C T Dewberry, K C Etchison, M M Seraffin, S A Peeples and S A Cooke J. Mol. Spectrosc. 251378 (2008)

[40] J A Shea and E J Campbell J. Chem. Phys. 815326 (1984)

[41] T Emilsson, T D Klots, R S Ruoff and H S Gutowsky J. Chem. Phys. 936971 (1990)

[42] P Thaddeus and M C McCarthy Spectrochim. Acta A Mol. Biomol. Spectrosc. 57757 (2001)

[43] V D Gordon, M C McCarthy, A J Apponi and P Thaddeus Astrophys. J. Supp. 138297 (2002)

[44] Y Sumiyoshi, Y Endo and Y Ohshima J. Mol. Spectrosc. 22222 (2003)

[45] K Seki, Y Sumiyoshi and Y Endo J. Chem. Phys. 1179750 (2002)

[46] R D Suenram and F J Lovas Astrophys. J. Lett. 342 L103 (1989)

[47] S G Batten, A G Ward and A C Legon J. Mol. Struct. 780-781 300 (2006)

[48] S Antolinez, A Lesarri, S Mata, S Blanco, J C Lopez and J L Alonso J. Mol. Struct. 612125 (2002)

[49] S A Cooke and M C L Gerry J. Mol. Spectrosc. 234195 (2005)

[50] J L Aonso, E J Cocinero, A Lesarri, S E Sanz and J C Lopez Angew. Chem. Int. Ed. 453471 (2006)

[51] E Arunan, P K Mandal, M Goswami and B Raghavendra Proc. Ind. Natl. Sci. Acad. 71A 377 (2005)

[52] M Goswami, P K Mandal, D H Ramadoss and E Arunan Chem. Phys. Lett. 39322 (2004)

[53] P K Mandal, D J Ramdass and E Arunan Phys. Chem. Chem. Phys. 72740 (2005)

[54] P K Mandal, M Goswami and E Arunan J. Indian Inst. Sci. 85 353 (2005)

[55] P K Mandal PhD Thesis (Indian Institute of Science: Bangalore) (2005)

[56] M Goswami and E Arunan Phys. Chem. Chem. Phys. 1314153 (2011)

[57] M Goswami and E Arunan J. Mol. Spectrosc. 268147 (2011)

[58] P Aiswaryalakshmi Ph.D Thesis (Indian Institute of Science, Bangalore) (2012)

[59] K I Peterson and W Klemperer J. Chem. Phys. 85725 (1986)

[60] M Goswami and E Arunan Phys. Chem. Chem. Phys. 118974 (2009)

[61] P K Mandal and E Arunan J. Chem. Phys. 1143880 (2001)

[62] B Raghavendra, P K Mandal and E. Arunan Phys. Chem. Chem. Phys. 85276 (2006)

[63] E Arunan et al. Pure Appl. Chem. 831637 (2011)

[64] D Mani, P Aishwaryalakshmi and E Arunan Asian J. Spectrosc. (Special Issue) 31 (2010)

[65] E Arunan, S Dev and P K Mandal Appl. Spectrosc. Rev. 39131 (2004)

[66] J Razec and P Hobza J. Chem. Theory Comput. 41835 (2008)

[67] G G Brown, B C. Dian, K O Douglass, S M Geyer, S T Shipman and B H Pate Rev. Sci. Instrum. 79053103 (2008) 